\title{
Improvements in Enzyme-Linked Photoassay Systems for Spatiotemporal Observation of Neurotransmitter Release
}

\author{
Kazunori Watanabe, Nobuto Takahashi, Naohiro Hozumi ${ }^{1}$ and Sachiko Yoshida* \\ Department of Environmental and Life Sciences, \\ Toyohashi University of Technology, 1-1 Hibarigaoka, Tempaku-cho, Toyohashi 441-8580, Japan \\ 'Int'l Cooperation Center for Engineering Education Development/ \\ Department of Electrical \& Electronic Information Engineering, \\ Toyohashi University of Technology, 1-1 Hibarigaoka, Tempaku-cho, Toyohashi 441-8580, Japan
}

(Received February 23, 2015; accepted September 25, 2015)

Key words: neurotransmitter, glutamate, GABA, enzyme immobilization, UV LED

Neurotransmitters and neuronal releasing molecules are not only the regulators of neuronal function but also the indicators of neuronal conditions. Glutamate and $\gamma$-amino butyric acid (GABA) play important roles in cerebellar differentiation and function. In the mature cortex, they are released from synapses and taken up by transporter molecules. We have developed enzyme-linked photoassay systems for glutamate, GABA, and adenosine triphosphate (ATP), and reported their release in the developing cerebellar cortex. Our systems showed slow transmitter release in the immature cerebellum, whereas it was hard to detect the fast synaptic release from mature neurons, because there were some limitations in time resolution and data depth derived from a charge-coupled device (CCD), and the enzyme-linked photodevice was sometimes unstable. In this study, we report the dynamic observation of neurotransmitter release in the developing cerebellar slices using improved photodevices and a high-speed 16-bit CCD. With this new system, the rapid measurement of transmitter release in a youngadult cerebellar cortex is possible. We suggest that these photoassay systems are useful for observing synaptic release in several diseases.

\section{Introduction}

Neurotransmitter molecules released from neurons are not only the regulators of neuronal transduction but also the indicators of neuronal conditions. ${ }^{(1-3)}$ Glutamate and $\gamma$-aminobutyric acid (GABA) are known as typical transmitters in the brain's cortex, and they play important roles as stimulators and suppressors, respectively. Lack of balance in the release of glutamate and GABA may lead to autism, epilepsy, or Parkinson's disease. . $^{(4)}$

${ }^{*}$ Corresponding author: e-mail: syoshida@ens.tut.ac.jp 
To observe spatiotemporal neurotransmitter release in the cerebellar cortex, we have recently developed an enzyme-linked photoassay system, which is a device with an immobilized enzyme on a quartz glass surface. Using this system, we observed glutamate or GABA release in developing cerebellar slices using either new or authorized methods. ${ }^{(5)}$ Enzyme-linked photoassay is sensitive and selective, and it can discriminate the substrates from their pharmacological analogues. Our system can detect transmitter release in the cerebral cortex, ${ }^{(6)}$ hippocampus, retina, and cultured cells, ${ }^{(7)}$ and made it possible to detect the release of adenosine triphosphate (ATP), ${ }^{(8)}$ glucose, sucrose, and fructose. On the other hand, enzymes tend to denature and separate from the quartz. For the detection of transmitter release in mature neuronal circuits, increasing the sensitivity and stability of the device is required.

In this paper, we propose new immobilizing methods and discuss the optimization of the enzyme-linked photoassay.

\section{Materials and Methods}

\subsection{Substrate and enzyme reaction}

Imaging neurotransmitter release was monitored for the reaction in which oxidoreductases generate reduced nicotinamide adenine dinucleotide $\left(\mathrm{NAD}^{+}\right)$or diphosphonucleotide $\left(\mathrm{NADP}^{+}\right)$. For glutamate, GABA, or adenosine triphosphate (ATP) imaging, we used glutamate dehydrogenase, GABA disassembly enzyme [GABase, Fig. 1(a)] or glyceraldehyde 3-phosphate dehydrogenase, respectively. ${ }^{(9-11)}$ The NADH or NADPH, the reductants of $\mathrm{NAD}^{+}$or $\mathrm{NADP}^{+}$, respectively, which is generated stoichiometrically, emits $480 \mathrm{~nm}$ fluorescence after excitation at $340-365 \mathrm{~nm}$.

\subsection{Surface photoexcitation}

For UV excitation, a quartz glass plate illuminated with an ultraviolet light-emitting diode (UV-LED, Nichia, Tokushima, Japan) was used. Leaking UV light onto the glass surface excited fluorescent NADH or NADPH [Fig. 1(b)].

\subsection{Imaging apparatus}

All fluorescence images through the inverted microscope (IX73, Olympus Co., Ltd., Tokyo, Japan) were observed by a cooled charge-coupled device (CCD) (ORCA-ER $\mathrm{CCD}$ ) or a high-speed complimentary metal-oxide semiconductor (CMOS) (ORCA-Flash 4.0) camera, supplied by Hamamatsu Photonics Co., Ltd., Hamamatsu, Japan. Imaging data were analyzed by iVision software (BD Biosciences, San Jose, CA, USA).

\subsection{Enzyme immobilization and sample preparation}

Enzymes were typically covalently immobilized on the quartz glass surface using a silane coupling agent and a crosslinking agent, 3-aminopropyltriethoxy silane (3-APTS) and glutaraldehyde, respectively [Fig. 2(a)].(12) These surface modifications determine both the stability of the enzyme reaction and the distance between the sample and the glass surface. 


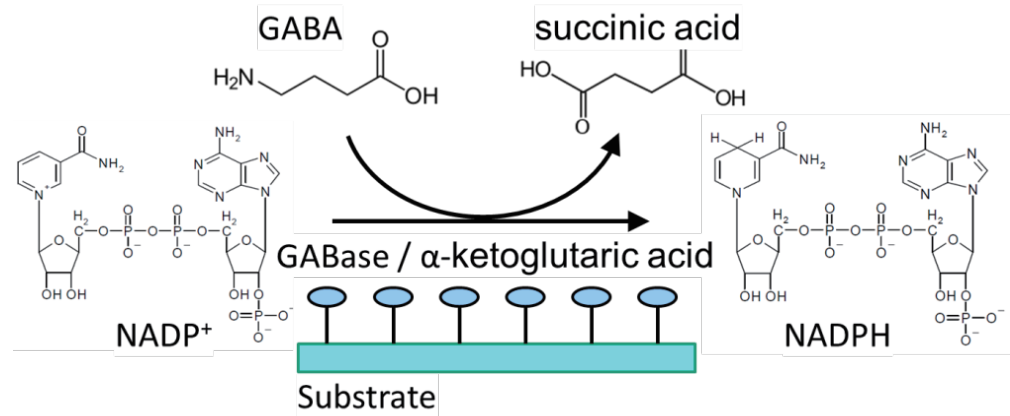

(a)

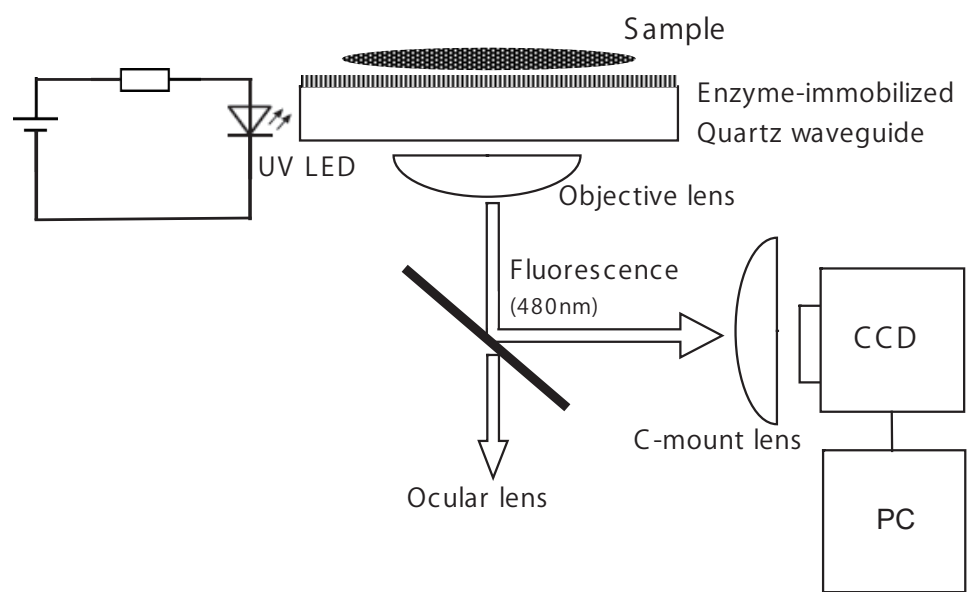

(b)

Fig. 1. (Color online) (a) Released neurotransmitters are oxidized by the oxidoreductase, and $\mathrm{NAD}(\mathrm{P}) \mathrm{H}$ is formed stoichiometrically. In GABA oxidization, released GABA is converted to succinic acid with NADPH formation by GABase. (b) Schematic diagram of the enzyme-linked photoassay system. The oxidoreductases are immobilized on the quartz glass surface, and the excitation light radiating from UV-LED passes through the quartz waveguide. Fluorescent images are obtained by CCD or CMOS, and analyzed using a computer system.

In some cases, glass surfaces were treated with either aromatic crosslinkers, 1,4-phenylene diisothiocyanate (1,4-DIC), or 1,3-phenylene diisothiocyanate [1,3-DIC, Fig. 2(b)], and glutaraldehyde (GA). ${ }^{(13)}$ Others were treated with a phosphonic acid, 11-aminoundecylphosphonic acid [11-AUPA, Fig. 2(c)], as a replacement for 3-APTS. ${ }^{(14)}$

Cerebellar acute slices were treated from postnatal day 3 (P3) to P15 in rats, sliced sagittally to a thickness of $400 \mu \mathrm{m}$ with a rotor slicer (Dohan EM, Kyoto, Japan), 


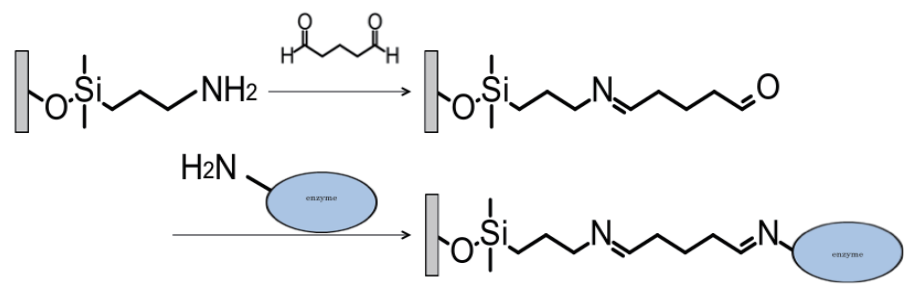

(a)

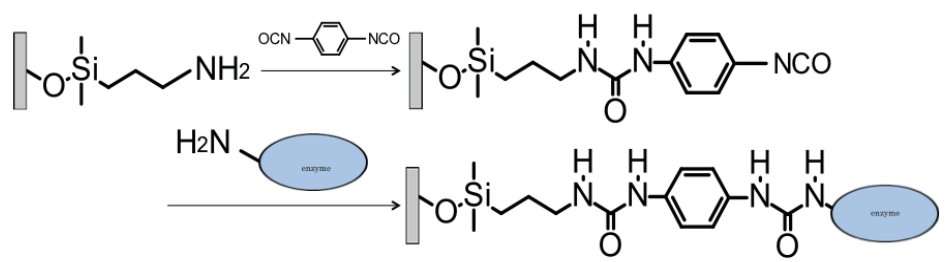

(b)

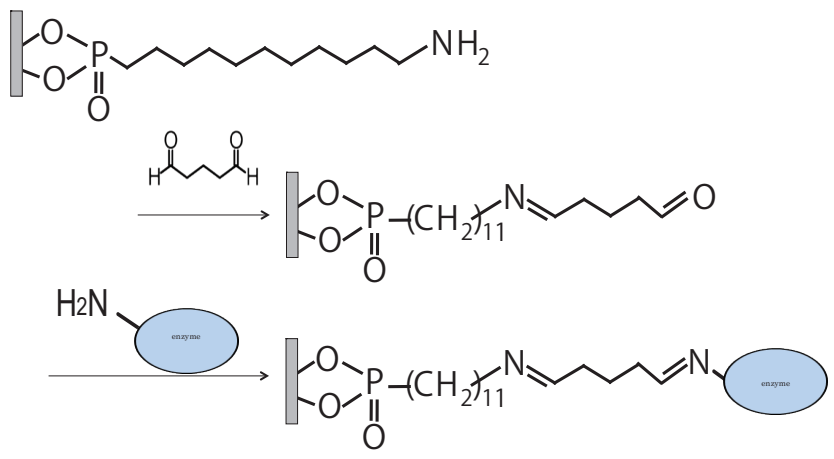

(c)

Fig. 2. (Color online) (a) Method of enzyme immobilization using 3-APTS and glutaraldehyde. Surface treatment and crosslinking between waveguide quartz and enzyme. (b) Crosslinking using 1,3-DIC. (c) Surface treatment with 11-AUPA.

and incubated in oxygen-aerated PBS for $45 \mathrm{~min}$. All experimental procedures were approved by the committee for the use of animals at Toyohashi University of Technology and by the guidelines of the Ministry of Education, Culture, Sports, Science and Technology, Japan. 


\section{Results}

\subsection{Spatiotemporal observation of glutamate release}

Figure 3(a) shows an illustration of rat cerebellar development. In the developing cerebellum, neuronal arrangement and circuit formation progress after birth. Granule cells, small input neurons, proliferate and migrate down from the external granular layer (EGL) to the internal granular layer (IGL). Purkinje cells, major output neurons, develop their dendrites and associate neuronal connections between granule cells and other interneurons. The layer of Purkinje cell somas is identified to be the Purkinje layer (PL). A neuronal circuit layer forms the molecular layer (ML). ${ }^{(15,16)}$ To understand the roles of neurotransmitters in the cerebellar development, we have developed a new visualizing device and, with it, we have observed spatiotemporal molecular dynamics.

Using the enzyme-linked photoassay system, we have observed many kinds of transmitter release in several developmental stages and organs. Our system has visualized both spontaneous and responsive transmitter release processes with $0.5 \mathrm{~s}$ time resolution. Figures $3(\mathrm{~b})-3(\mathrm{k})$ show the transitions in glutamate release in response to $100 \mu \mathrm{M}$ GABA application in developing cerebellar slices. ${ }^{(17)}$ Glutamate was released in both the EGL and the IGL, whereas the PL was indicated by a negative line. In the developing cerebellum, the granule cells that distributed in the EGL and IGL are the only neurons that release glutamate, so both layers showed fluorescence activities. Glutamate release in P3 cerebellar slices appeared in both layers slowly but continuously, whereas it started rapidly in the lower EGL and then spread to the IGL within a short time in P7 celebellar slices. ${ }^{(18)}$ Granule cells in the P3 cerebellum did not develop sufficiently to react to GABA stimulation nor release the transmitter actively, but they still proliferated. On the other hand, the granule cells in the P7 cerebellum developed sufficiently to react to GABA stimulation, so they released glutamate rapidly.

Although spatiotemporal observation could give us dynamic information about neuronal reaction, our system needs to be improved in terms of stability, sensitivity and time resolution for us to observe fast synaptic transmissions. The targets of our improvements were the (1) sensing CCD, (2) excitation waveguide, and (3) manner of enzyme immobilization shown in Fig. 2.

\subsection{Effects of new crosslinkers and surface treatment}

Two types of glass devices with either aromatic crosslinkers, 1,3-DIC or 1,4-DIC, and GA were examined to observe spontaneous GABA release with $500 \mathrm{~ms}$ time resolution using ORCA ER CCD. The device formed using 1,3-DIC and GA gave images with a better contrast of GABA release than the GA crosslinked device in the P10 cerebellar slice [Fig. 4(a)], whereas it showed no difference in the P6 cerebellar slice. The 1,4-DIC crosslinked device yielded no good images.

The aromatic crosslinkers make the glass surface hydrophobic. Because mature brain tissues become hydrophobic as the myelin structure develops, 1,3-DIC crosslinking should increase the affinity of the enzyme for the tissues.

The binding between the glass and the acceptor molecules has been weak, because the silane coupling agents tend to undergo hydrolysis under biological conditions. The 
(a)
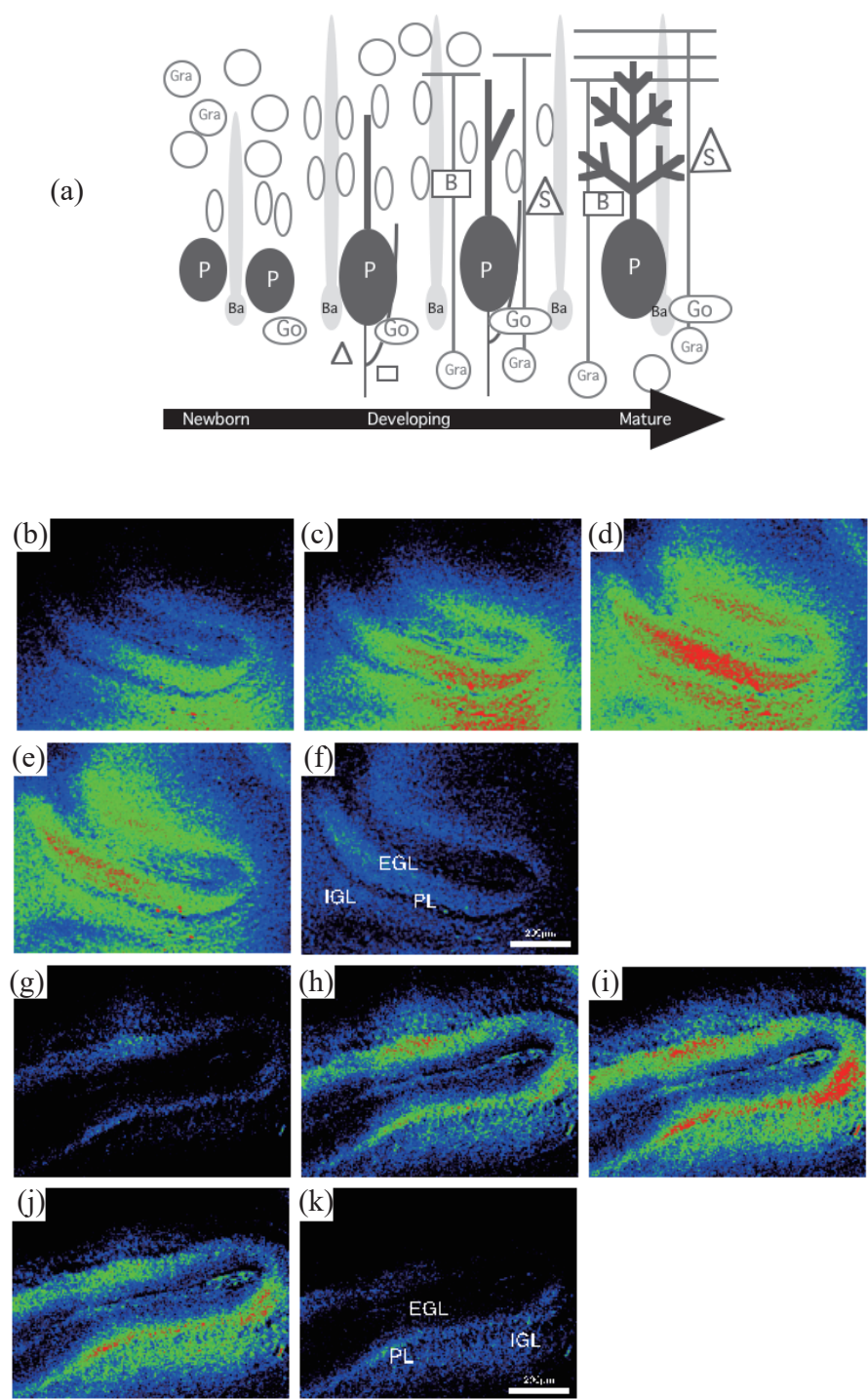

Fig. 3. (a) Diagram of cerebellar development. In the newborn cerebellum (1 to 3 days after birth), granule cell progenitors (Gra) proliferate in the EGL, while immature Purkinje cells (P) form the PL with Golgi cells (Go) and some Bergmann glia (Ba). During the developement, 5 to 7 days after birth, Gra-cells elongate their axon and migrate inside, and P-cells spread their dendrites and connect to other neurons within two weeks. B denotes basket cells, and S, satellite cells. Evoked glutamate wave with GABA application in developing cerebellar cortex. (b)-(f): 2.0, 4.5, 7.0, 12.5, and $23.5 \mathrm{~s}$ after stimulation in $\mathrm{P} 3$ cerebellar cortex, respectively. (g)-(k): 0.5, 2.5, 4.0, 11.0, and $16.0 \mathrm{~s}$ after stimulation in $\mathrm{P} 7$ cerebellar cortex, respectively. 


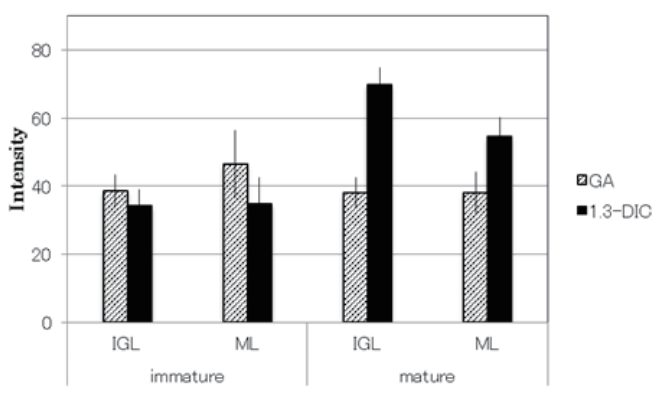

(a)

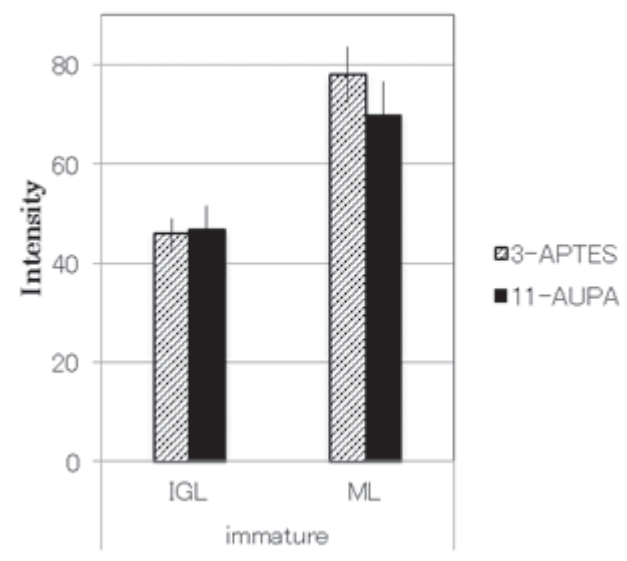

(b)

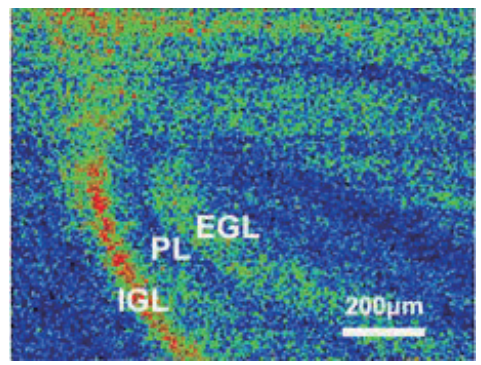

(c)

Fig. 4. (Color online) (a) New crosslinker, 1,3-DIC, gave us better contrast images than GA in mature cerebellar observation, while it made little difference from GA in immature organ. (b) The 11-AUPA-GA treatment showed the same result as the APTS-GA treatment in the immature organ. (c) Spontaneous GABA release image in P12 cerebellar slice using 1,3-DIC crosslinking glass device.

surface treatment by 11-AUPA, as a replacement for 3-APTS, was expected to inhibit hydrolysis, but it had low affinity for the glass. We constituted a new glass device with 11-AUPA-coupling enzymes and examined its sensitivity and stability. Figure 4(b) shows that the new device performed with the same sensitivity and stability as the device with APTS.

\subsection{Observation using high-speed CMOS camera}

The fluorescence intensity of NADH is very low and is only a few thousands of the intensity of typical artificial fluorescence. To collect data with sufficient time resolution, a highly sensitive and rapid data transferring camera is required. The time resolution 
shown in Fig. 3 is $0.5 \mathrm{~s}$ for the 12-bit ORCA CCD, which is too low to detect the synaptic transmitter reaction.

A 16-bit CMOS camera, Flash 4.0, could detect weak light and transfer data in less than a microsecond. Using this camera, transient glutamate release could be detected with a $20 \mathrm{~ms}$ time resolution (Fig. 5). In developing the P7 cerebellum, glutamate release was increased in the EGL by applying a glutamate receptor-stimulating agent, $\alpha$-amino-3-hydroxy-5-methyl-4-isoxazolepropionic acid (AMPA). Even in the premature P14 cerebellum, the increment in the rate of glutamate release was observed. It was not strong and noiseless, but the AMPA stimulation-induced glutamate release was observed in the ML and IGL where the glutamatergic neurons are distributed.

\section{Discussion}

The detection of neurotransmitter release gives us important information about developmental conditions and diseases. Parkinson's disease, a degenerative disorder of the central nervous system, is caused by the alteration of the release of neurotransmitters. The detection of the spatial or temporal alteration of the release would require early diagnosis and treatment of Parkinson's disease. In immature or lesioned neuronal organs, transmitters are released and taken up slowly, so the time resolution required is from 0.5 to $1 \mathrm{~s}$. In young-adult stages, the release speed becomes higher than that in the immature stage within $20 \mathrm{~ms}$.
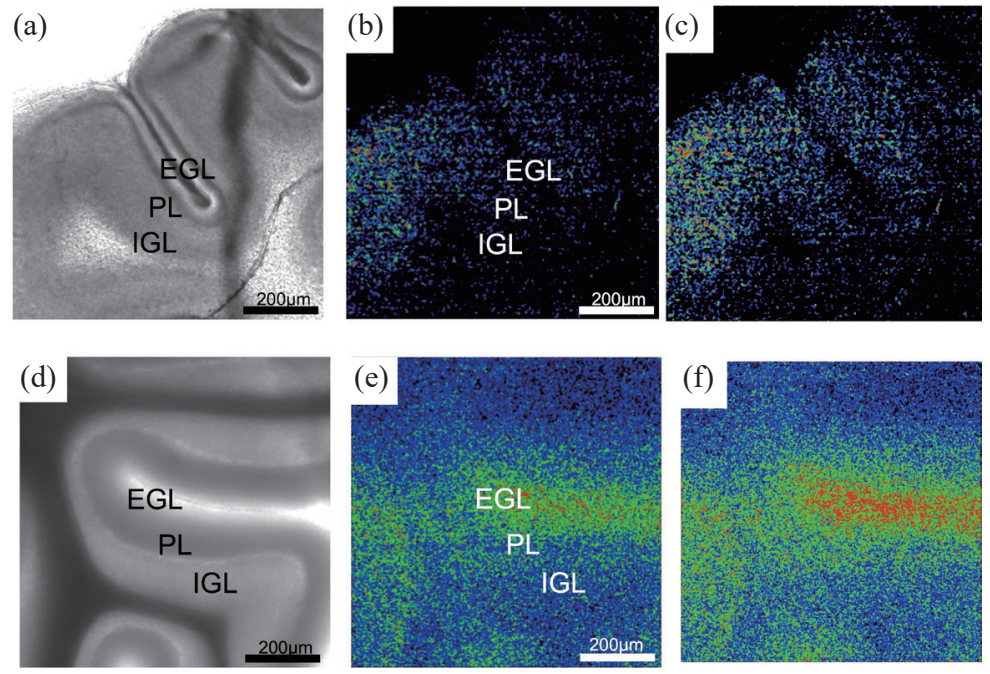

Fig. 5 (Color online) Evoked glutamate release images to AMPA stimulation for $20 \mathrm{~ms}$ time resolution using Flash 4.0 CMOS system. (a)-(c) P7 cerebellar slice; (d)-(f) P14 cerebellar slice. (a) and (d) Phase contrast light images. (b) and (e) Fluorescence images before stimulation and (c) and (f) just after AMPA stimulation. 
Enzyme-linked assays were applied previously for chemical detection because of their specificity. In spatial observations, however, their fluorescence intensity is too weak to detect. Our enzyme-linked photodevice was developed to detect spatiotemporal neurotransmitter release, and it was improved to observe rapid synaptic release. New crosslinkers could contribute to a more sensitive detection, and the phosphonic surface treatment would expand the range of applications. In order to detect a highspeed transmitter release, both the light accumulation system for weak fluorescence and the close contact between the specimen and the enzyme are required. At present, our photodetection system detects several ms releases from neuronal synapses in the presence of noise, and in the future, it could give us more noiseless observations using an optimal image processing system.

\section{Conclusions}

The newly developed enzyme-linked photoassay is useful for the visualization of neurotransmitter release in brain slices. In the immature cerebellum, the granule cells release glutamate slowly or rapidly at their stage of neuronal development and synaptogenesis.

Using a fast new system, the rapid measurement of transmitter release in a youngadult cerebellar cortex became possible. Crosslinkers and other device techniques are required for stable observations. We suggest that the photoassay systems have advantages for the observation of synaptic release in several diseases.

\section{Acknowledgements}

This study was supported by grants from Scientific Research (C) 23500516 and 26350498, and Health Labor Sciences Research.

\section{References}

1 J. Moran and M. Rivera-Gaxiola: J. Neurosci. Res. 33 (1992) 239.

2 P. Varju, Z. Katarova, E. Madarasz and G. Szabo: Cell Tissue Res. 305 (2001) 239.

3 D. Casel, J. Brockhaus and J. W. Deitmer: J. Physiol. 568 (2005) 111.

4 M. Ochi, S. Shiozaki and H. Kase: Neuroscience 127 (2004) 223.

5 T. Morishima, M. Uematsu, T. Furukawa, Y. Yanagawa, A. Fukuda and S. Yoshida: Neurosci. Res. 67 (2010) 347.

6 T. Wang, T. Kumada, T. Morishima, S. Iwata, T. Kaneko, Y. Yanagawa, S. Yoshida and A. Fukuda: Cereb Cortex 24 (2014) 1088.

7 S. Yoshida and N. Hozumi: JNNS 20 (2013) 14.

8 H. Muramoto, Y. Sekino, N. Hozumi, A. Fukuda and S. Yoshida: 42th Neuroscience Meeting (2012) (CD-ROM).

9 B. Innocenti, V. Parpura and P. G. Haydon: J. Neurosci. 20 (2000) 1800.

10 M. Israel and B. Lesbats: J. Neurochem. 67 (1996) 2624.

11 S. Yoshida: Japanese Patent No. 4480952 (2010).

12 L. Blasi, L. Longo, PP. Pompa, L. Manna, G. Ciccarella, G. Vasapollo, R. Cingolani, R. Rinaldi, A. Rizzello, R. Acierno, C. Storelli and M. Maffia: Biosens. Bioelectron. 21 (2005) 30 . 
13 N. Aissaoui, J. Landoulsi, L. Bergaoui, S. Boujday and J. F. Lambert: Enzyme Microb. Technol. 52 (2013) 336.

14 M. Bariana, M. S. Aw, M. Kurkuri and D. Losic: Int. J. Pharm. 443 (2013) 230.

15 M. Yamada, M. Terao, T. Terashima, T. Fujiyama, Y. Kawaguchi, Y. Nabeshima and M. Hoshino: J. Neurosci. 27 (2007) 10924.

16 M. Ito: The Cerebellum and Neural Control (Raven Press, New York, 1984) Chap. 1.

17 M. L. Fiszman and A. Schousboe: J. Neurosci. Res. 76 (2004) 435.

18 K. Okochi, S. Hirano, K. Maruya, T. Morishima, C. Takayama, A. Fukuda and S. Yoshida: Neurosci. Res. 61 (2008) S114.

\begin{abstract}
About the Authors
Kazunori Watanabe was born in Hokkaido, Japan, on February 3, 1992. He is a 2nd-year student in the Graduate School of Toyohashi University of Technology. His research focuses on the optimization of surface modifications for biosensors and the detection of neurotransmitters. He is a member of the Japan Neuroscience Society.
\end{abstract}

Nobuto Takahashi was born in Tokyo, Japan, on December 9, 1992. He is a 1st-year student in the Graduate School of Toyohashi University of Technology. His research focuses on bioengineering photoanalysis of cells and the developmental mechanism of cultured neurons. He is a member of the Japan Neuroscience Society.

Naohiro Hozumi was born in Kyoto, Japan, on April 2, 1957. He received his B.S., M.S., and Ph.D. degrees in 1981, 1983, and 1990, respectively, from Waseda University. He was at the Central Research Institute of Electric Power Industry (CRIEPI) from 1983 to 1999 . He was an associate professor at Toyohashi University of Technology from 1999 to 2006, and a professor at Aichi Institute of Technology from 2006 to 2011. Since 2011, he has been a professor at Toyohashi University of Technology. He has been engaged mostly in research on insulating materials and diagnosis of high-voltage equipment and acoustic measurement for biological and medical applications. In 1990 and 1999, he received awards from IEE of Japan for his outstanding research papers. He is a member of IEEE, IEE of Japan, and the Acoustic Society of Japan.

Sachiko Yoshida was born in Toyama, Japan, on January 24, 1961. She received her B.S., M.S., and Ph.D. degrees in 1983, 1986, and 1990, respectively, from the University of Tokyo. She was a JSPS Postdoctoral Researcher from 1990 to 1992, a JST PRESTO Researcher from 1992 to 1994, and a research associate at Toyohashi University of Technology from 1995 to 1996. Since 1996, she has been a lecturer at Toyohashi University of Technology. Her research interests focus on physiological interaction and morphological transformation through brain differentiation, and their detection. She is a member of IEEE, International Brain Research Organization, the Society for Neuroscience, and the Japan Neuroscience Society. 